\title{
Factors associated with fluctuations in repeated measurements of intraocular pressure using the Goldmann applanation tonometer in Japanese patients with primary open-angle glaucoma
}

This article was published in the following Dove Press journal:

Clinical Ophthalmology

\author{
Kiyoshi Yaoeda ${ }^{1,2}$ \\ Atsushi Fukushima' \\ Motohiro Shirakashi ${ }^{3}$ \\ Atsushi Miki ${ }^{4}$ \\ Takeo Fukuchi ${ }^{2}$ \\ 'Department of Ophthalmology, \\ Yaoeda Eye Clinic, Nagaoka, Niigata, \\ Japan; ${ }^{2}$ Division of Ophthalmology and \\ Visual Sciences, Niigata University \\ Graduate School of Medical and \\ Dental Sciences, Niigata, Niigata, \\ Japan; ${ }^{3}$ Department of Ophthalmology, \\ Kido Eye Clinic, Niigata, Niigata, \\ Japan; ${ }^{4}$ Department of Ophthalmology, \\ Kawasaki Medical School, Kurashiki, \\ Okayama, Japan
}

Purpose: The aim of this study was to determine whether fluctuations in intraocular pressure (IOP) occur as a result of the order of IOP measurements or successive IOP measurements in patients with glaucoma and, if so, identify the factors causing these fluctuations.

Patients and methods: Four hundred twenty-eight eyes of 214 Japanese patients with primary open-angle glaucoma (POAG) were enrolled. Patients treated with beta-blockers or prostaglandin analogs alone were included. Additionally, in the IOP measurements by noncontact tonometer, the same cases of IOP of the right and left eyes prior to this study were included in this study. Four successive IOP measurements were carried out using a Goldmann applanation tonometer as follows: IOP was measured in the first eye (right or left) and then in the fellow eye and IOP was again measured in the first eye and then in the fellow eye. Repeated-measures analysis of variance was used to test the differences in IOP between successive measurements. Generalized linear mixed models were used to test differences in IOP measurements between the right and the left eyes on repeated applanation tonometry and according to the order of measurement. Conditional binomial logistic regression analysis was used to identify factors associated with fluctuating repeated applanation tonometry measurements. A $P$-value of $<0.05$ was considered statistically significant.

Results: IOP values decreased significantly according to the number of measurements (13.8-13.0; $P<0.001-0.036$, respectively). There was no significant difference in IOP measurements between the right and left eyes. The first IOP measurement was significantly higher than the fourth measurement $(P=0.038)$; however, there was no significant difference between other combinations. The use of a prostaglandin analog was the only significant contributor to fluctuating IOP measurements $(P=0.002)$.

Conclusion: IOP measured in the first eye, either right or left, was higher than that measured in the fellow eye in Japanese patients with POAG. The use of a prostaglandin analog may be associated with fluctuating IOP on repeated applanation tonometry.

Keywords: Goldmann applanation tonometer, measurement order, right and left eyes, glaucoma, intraocular pressure

\section{Introduction}

Glaucoma is a disease accompanied by changes in the optic nerve called glaucomatous optic neuropathy and corresponding visual field abnormality. ${ }^{1}$ Currently, the only treatment based on the evidence to stop or delay the progression of glaucomatous optic neuropathy and glaucomatous visual field deterioration is decreasing 
the intraocular pressure (IOP). ${ }^{1}$ Therefore, accurate IOP measurement is indispensable for the diagnosis and treatment of glaucoma. The IOP measurement using the Goldmann applanation tonometer (GAT) developed by Goldmann and Schmidt ${ }^{2}$ in 1957 is currently regarded as one of the standard examinations for glaucoma management. However, it is known that IOP measurements by GAT tend to fluctuate because of several artifactual factors, including squeezing of the eyelids and the Valsalva maneuver. ${ }^{3-6}$ Understanding and eliminating these artifactual factors are important for the accurate diagnosis and management of patients with glaucoma.

In an analysis of the Ocular Hypertension Treatment Study, the mean IOP measured in the right eye was $0.3 \mathrm{mmHg}$ higher than that measured in the left eye. ${ }^{7}$ Other studies that measured the right eye first also reported that the IOP measured in the right eye was higher than that in the left eye, ${ }^{8-10}$ while some studies have reported higher IOP on the left eye. ${ }^{11}$ Bhorade et al attributed this discrepancy to the order in which IOP measurements were taken (ie, right eye measured first) because of the slight decrease in IOP associated with successive IOP measurements. ${ }^{7}$ Whether this difference in IOP reflects a true physiologic difference between the right and left eyes or an artifactual difference related to the right eye being measured first needs to be investigated. A recent study in healthy volunteers found that IOP measured in the first eye, either right or left, was higher than the IOP subsequently measured in the fellow eye. ${ }^{12}$ The authors of that report suggested that the higher IOP measurements in the right eyes were most likely attributable to the right eyes being measured first rather than a physiologic difference in IOP between the eyes. However, in a study of repeated applanation tonometry, a decrease in IOP was documented in subjects with glaucoma but not in those without glaucoma. ${ }^{13}$ These fluctuations in IOP measurements may reflect artifactual factors; however, it is not known whether other ocular conditions or patient factors are involved.

The purpose of this study was to determine whether the order of IOP measurement or repeated applanation tonometry affects IOP measurements in patients with glaucoma and, if so, identify the factors causing fluctuations in IOP.

\section{Patients and methods}

In the present study, all acquired data were obtained from routine examination in Yaoeda Eye Clinic. Additionally, since any intervention tests were excluded in this study, we did not contact a research ethics committee. As the authors had no access to a research ethics committee, the study adhered to the tenets of the Declaration of Helsinki. All patients included in the study gave their informed consent to undergo the IOP examinations. The written informed consents were obtained from all participants in this study. All study participants were Japanese, had primary open-angle glaucoma (POAG), and were recruited from the outpatient section of Yaoeda Eye Clinic. POAG was defined as glaucomatous optic disk damage and abnormal visual field test results with a normal anterior chamber angle. Signs of glaucomatous optic disk damage included diffuse or localized loss of the neuroretinal rim, excavation, and defects in the retinal nerve fiber layer. An abnormal visual field was defined as a pattern SD outside the normal 95\% confidence limits or a glaucoma hemifield test result that was outside normal limits using the Swedish interactive thresholding algorithm (SITA) standard 24-2 testing protocol with a Humphrey field analyzer 750 (Carl Zeiss Meditec AG, Jena, Germany). All patients underwent a routine ophthalmic examination or a review of their most recent ophthalmic examination before inclusion in the study. Patients undergoing treatment with a beta-blocker or prostaglandin analog alone were included to simplify the analysis of the study findings. Additionally, in the IOP measurements by noncontact tonometer (NCT) using a CT-90A system (Topcon Corporation, Tokyo, Japan) of both eyes in random order, the same cases of IOP of the right and left eyes prior to this study were included in this study. Patients with any corneal pathology (eg, corneal scarring, edema, epithelial defect, and grafts) or corneal astigmatism greater than $3 \mathrm{D}$ were excluded, as were patients who had undergone cataract or glaucoma surgery.

The patients were randomly divided into the following two groups: IOP was measured in the right eye in one group and IOP was measured in the left eye in the other group. Four successive IOP measurements were obtained by measuring the IOP in the first eye and then in the fellow eye (first and second measurements, respectively, defined as the first cycle) and by measuring the IOP again in the first eye and the fellow eye (third and fourth measurements, respectively, defined as the second cycle).

A calibrated GAT mounted on a slit-lamp biomicroscope (Haag-Streit Co., Bern, Switzerland) was used for the IOP measurements for analysis in the present study. One drop of $0.4 \%$ benoxinate hydrochloride was instilled in both eyes prior to the measurement of IOP. The drop was instilled in each eye in the same sequence as the order of IOP measurement (eg, if the right eye was measured first, the fluorescein was applied to the right eye first). Following local anesthesia, a fluorescein strip was applied to each eye. To avoid interobserver variability, ${ }^{12,13}$ all measurements were performed by 
a single experienced examiner $(\mathrm{KY})$; all IOP readings were performed by other examiners.

Paired $t$-tests were used to compare the characteristics of the right eyes with those of the left eyes. Repeatedmeasures analysis of variance was used to test differences in IOP between the successive measurements (first to fourth). Generalized linear mixed models were used to test differences in repeated applanation tonometry (first to fourth measurements) with laterality of the eye as a random factor or between the right and left eyes with the order of successive measurements as a random factor. Factors associated with fluctuations in IOP values obtained by repeated applanation tonometry were tested for significance by conditional binomial logistic regression analysis.

The statistical analyses were performed using the SPSS Statistics Version 20.0 software (IBM Corporation, Armonk, NY, USA) and the MedCalc version 11 program (MedCalc Software bvba, Mariakerke, Belgium). A $P$-value of $<0.05$ was considered statistically significant.

\section{Results}

Four hundred twenty-eight eyes of 214 patients with POAG were enrolled in the study. The patient characteristics are shown in Table 1 . The mean age of the study participants (121 females and 93 males) was 70.2 years. The average NCT measurement was $14.7 \mathrm{mmHg}$ in both eyes. Eighty-one patients were taking a beta-blocker, and 133 patients were taking a prostaglandin analog.

IOP was measured in the right eye in 109 patients and in the left eye in 105 patients. There was no statistically significant difference in refraction, cylinder, corneal curvature, mean deviation determined by the SITA standard 24-2 testing protocol, or IOP measurements obtained in the first and second cycles between the right and left eyes (Table 2).

Table I Patient characteristics $(n=2 \mid 4)$

\begin{tabular}{ll}
\hline Age (years) & $70.2 \pm 1 \mathrm{I} .9$ (32-94) \\
Sex, female (\%) & $121(57)$ \\
$\mathrm{NCT}(\mathrm{mmHg})$ & $14.7 \pm 2.8(10-22)$ \\
Timolol & 74 patients \\
Carteolol & 7 patients \\
Beta-blockers & 81 patients \\
Latanoprost & 99 patients \\
Travoprost & 28 patients \\
Tafluprost & 4 patients \\
Bimatoprost & 2 patients \\
Prostaglandin analogs & 133 patients \\
\hline
\end{tabular}

Notes: The data are shown as the mean \pm standard deviation (range). NCT is used to measure intraocular pressure.

Abbreviation: $\mathrm{NCT}$, noncontact tonometer.
IOP decreased significantly with successive measurements (from 13.8 to $13.0 \mathrm{mmHg} ; P<0.001-0.036$; Table 3 ). Generalized linear mixed models revealed no statistically significant difference in IOP between the right and left eyes. The first IOP measurement was significantly higher than the fourth IOP measurement $(P=0.038)$. However, no significant differences were found for other combinations of measurements. The IOP was high in 97 patients on the first measurement and low in eight patients on the fourth measurement; the same value was recorded on both the first and fourth measurements in 109 patients. Ninety-seven cases in which the first IOP measurement was high $(=0)$ and 117 other cases $(=1)$ were defined as binary dummy objective variables, and conditional logistic regression analysis was performed using age, sex, use of a prostaglandin analog $(=0$, use of a betablocker $=1$ ), refraction, cylinder, corneal curvature, and mean deviation. Only use of a prostaglandin analog was identified as a factor contributing to fluctuating IOP measurements ( $P=0.002$; Table 4).

\section{Discussion}

In this study of eyes in patients with POAG, IOP measured by GAT decreased with successive measurements independent of laterality, as previously reported in healthy volunteers. ${ }^{12}$ IOP was measured initially higher as opposed to fellow eyes, and IOP decrease was measured with repeated applanation tonometry. Pekmezci et al ${ }^{12}$ suggested that one plausible explanation for earlier IOP measurements causing artifactually higher IOPs than those measured later was patients' lack of familiarity with tonometry and anxiety during the early measurements. Another study reported similar findings that IOP was $2 \mathrm{mmHg}$ lower in patients who underwent sham tonometry prior to real tonometry than patients who did not, suggesting that familiarity with applanation tonometry may result in a lower measured IOP. ${ }^{14}$

Squeezing of the eyelids or other unknown phenomena associated with anxiety or unfamiliarity with tonometry may play a role in the higher initial IOP measurements. Attempted eyelid closure has been associated with increased IOP measurement by GAT in normal volunteers ${ }^{15}$ and in patients with glaucoma. ${ }^{16}$ Patient factors, such as the Valsalva maneuver and squeezing of the eyelids, are known to elevate IOP. ${ }^{3-6}$ Pekmezci et al ${ }^{12}$ confirmed that IOP measurements associated with moderate or severe ocular squeezing are higher than those associated with no or mild ocular squeezing. Furthermore, they found that moderate or severe ocular squeezing occurred more frequently during earlier IOP measurements and decreased with repeated applanation tonometry. 
Table 2 Comparison of right and left eyes

\begin{tabular}{llll}
\hline & Right eye & Left eye & P-value* \\
\hline Refraction (D) & $-1.34 \pm 3.67(-13.25$ to 4.25$)$ & $-1.34 \pm 3.59(-13.75$ to 4.25$)$ & 0.238 \\
Cylinder (D) & $-1.09 \pm 0.64(-2.75$ to 0$)$ & $-1.10 \pm 0.74(-2.75$ to 0$)$ & 0.875 \\
Corneal curvature $(\mathrm{mm})$ & $7.66 \pm 0.25(6.90$ to 8.39$)$ & $7.67 \pm 0.27(6.84$ to 8.45$)$ & 0.764 \\
Mean deviation** $(\mathrm{dB})$ & $-3.40 \pm 3.99(-17.68$ to 1.58$)$ & $-3.46 \pm 3.89(-17.36$ to 1.44$)$ & 0.806 \\
IOP on first cycle $(\mathrm{mmHg})$ & $13.7 \pm 2.8(8$ to $2 \mathrm{I})$ & $13.3 \pm 2.8(7$ to 21$)$ & 0.188 \\
IOP on second cycle $(\mathrm{mmHg})$ & $13.2 \pm 2.7(7$ to $2 \mathrm{I})$ & $13.1 \pm 2.9(7$ to 21$)$ & $0.54 \mathrm{I}$ \\
\hline
\end{tabular}

Notes: The data are shown as the mean \pm standard deviation (range). The IOP values were obtained with SITA standard 24-2 testing protocol using a Humphrey field analyzer 750 . *Paired $t$-test. **A $P$-value of $<0.05$ was considered statistically significant.

Abbreviations: IOP, intraocular pressure; SITA, Swedish interactive thresholding algorithm.

These observations suggest that decreased IOP measurements with successive applanation tonometry may be partially related to decreased ocular squeezing during repeated procedures. $^{12}$

Another factor that may explain why IOP measurement decreases with repeated applanation tonometry is the changing status of the tear film, in that a smaller tear meniscus is associated with underestimation of IOP..$^{17,18}$ A previous study that included performing tonography in one eye reported a decrease in the IOP in the fellow eye, likely because of evaporation of the tear film. ${ }^{19}$ Evaporation of the tear film over time may occur naturally or because of increased contact time with a topical anesthetic and fluorescein and may cause fluorescein "quenching" resulting in hypofluorescence and an underestimation of the IOP. ${ }^{17,18}$ Both increased tear film evaporation and fluorescein quenching have a greater effect over time and may be related to the decrease in IOP seen with repeated applanation tonometry. To exclude these effects in the present study, the drop was instilled in each eye in the same sequence as the order of IOP measurement.

Another factor that may help to explain the initially increased IOP is manipulation of the eyelid by the examiner at the time of measurement. The mechanism of elevation of IOP as a result of eyelid manipulation can be explained in several ways. First, in the process of manipulating the eyelids, digital pressure is also applied to the orbit. As a result, the soft tissue around the eyeball is compressed, which increases the ocular venous pressure. ${ }^{20,21}$ Second, it would also be expected

Table 3 Comparison of successive IOP measurements

\begin{tabular}{lll}
\hline Measurement & IOP $(\mathbf{m m H g})$ & $P$-value* \\
\hline First & $13.8 \pm 2.8(8-2 \mathrm{I})$ & \\
Second & $13.3 \pm 2.7(7-2 \mathrm{I})$ & 0.000 (first vs second) \\
Third & $13.2 \pm 2.7(7-2 \mathrm{I})$ & 0.036 (second vs third) \\
Fourth & $13.0 \pm 2.7(7-2 \mathrm{I})$ & 0.001 (third vs fourth) \\
\hline
\end{tabular}

Notes: The data are shown as the mean \pm SD (range). *Repeated-measures analysis of variance.

Abbreviation: IOP, intraocular pressure. that manipulation of the eyelid itself could compress the eyeball, thereby directly increasing IOP ${ }^{20}$ Third, The patient's anxiety or unfamiliarity with tonometry might decrease on repeated applanation tonometry, resulting in decreased ocular squeezing, a decrease in the digital pressure exerted by the examiner, and a decrease in the IOP measurement.

In a study that included both patients with glaucoma and normal volunteers, Gaton et $\mathrm{al}^{13}$ reported that eyes with glaucoma showed a significant decrease in IOP on repeated applanation tonometry measurements relative to the first measurement, whereas control eyes did not. It is possible that each repeated applanation with mechanical pressure on the cornea in eyes with open-angle glaucoma causes more distortion and greater widening of the anterior chamber angle, resulting in additional drainage of aqueous fluid and, consequently, a further decline in IOP. ${ }^{13}$ The findings of our study may reflect these mechanisms. If this hypothesis is true, IOP fluctuations in repeated measurements may decrease in patients who have undergone cataract surgery ${ }^{22}$ or glaucoma surgery. ${ }^{23}$

In the present study, use of a prostaglandin analog was the only factor associated with fluctuation of IOP measurements on repeated applanation tonometry. Peplinski and Albiani Smith ${ }^{24}$ first reported "deepening of the upper eyelid sulcus (DUES)" as a side effect of prostaglandin F2 $\alpha$ analogs in

Table 4 Regression analysis to determine factors associated with fluctuating intraocular pressure measurements

\begin{tabular}{llll}
\hline Variables & $\begin{array}{c}\text { Odds } \\
\text { ratio }\end{array}$ & $\mathbf{9 5 \%} \mathbf{C l}$ & P-value* \\
\hline Age & 0.994 & $0.987-I .002$ & 0.129 \\
Sex & 0.633 & $0.360-I .114$ & 0.113 \\
Use of a prostaglandin analog & 2.366 & $1.364-4.105$ & 0.002 \\
Refraction & 0.958 & $0.775-I .185$ & 0.693 \\
Cylinder & 1.394 & $0.882-2.205$ & 0.155 \\
Corneal curvature & 0.426 & $0.016-11.466$ & 0.612 \\
Mean deviation & 1.052 & $0.969-1.141$ & 0.225 \\
\hline
\end{tabular}

Note: *Conditional binomial logistic regression analysis. 
users of bimatoprost in 2004. DUES has since become a commonly recognized cosmetic side effect of prostaglandin F2 $\alpha$ analogs. However, the term "DUES" refers to upper eyelid problems only and similar side effects in the lower eyelids should be considered. Recently, "prostaglandinassociated periorbitopathy (PAP)" was proposed as a more general term for side effects of prostaglandin F $2 \alpha$ analogs that occur around the eyelids. ${ }^{25}$ PAP includes ptosis of the upper eyelid, DUES, involution dermatochalasis, atrophy of orbital fat, mild enophthalmos, flattening of the lower eyelid bags, inferior scleral show, and a tight orbit. As tight orbit syndrome, IOP can be overestimated because of the structural changes in the eyelids, and orbits reported to occur during long-term use of prostaglandin agents by patients with glaucoma who developed PAP. ${ }^{26,27}$ The decrease in IOP on successive measurements may be attributed to both the examiner and the subject who became more relaxed during opening of the eyelids.

There are several limitations to this study. First, there is a possibility that the true IOP may have been different between the right and the left eyes, though the same case of IOP as measured by NCT of both eyes was included in this study. Therefore, the fourth IOP measurement was higher in eight patients than the first IOP measurement in this study. Second, an effect of race cannot be excluded. Unlike in the eyes of Caucasian individuals, eyes in Asian individuals have a small palpebral fissure height, so the measurement of IOP often requires manual manipulation of the eyelids. ${ }^{28,29}$ All the patients in our study were Japanese and had POAG, so manual manipulation of the eyelids was necessary in all cases. A further study is needed to investigate the influence of race on variation in successive IOP measurements. Third, it was not possible to examine several other variables that might affect IOP measurements, such as central corneal thickness, corneal hysteresis, and axial length, because these variables are not measured as part of routine ophthalmic examinations at our facility. A further study is needed to determine the effect of these variables on successive IOP measurements with repeated applanation tonometry. Fourth, interobserver variability was not investigated in this study. As many similar studies were being measured by a single examiner to avoid interobserver variability, ${ }^{12,13}$ all measurements were performed by a single examiner in this study. Studies have shown that in $20 \%-30 \%$ of patients, the interobserver variability of IOP measurements with the GAT may be as high as $\geq 2 \mathrm{mmHg} .{ }^{30,31}$ Further studies are needed to investigate the influence of intra- or interobserver variabilities on the repeated IOP measurements.

\section{Conclusion}

In this study, IOP measurements fluctuated according to the order of IOP measurement and successive IOP measurements in Japanese patients with POAG. The IOP measured in the first eye, either right or left, was higher than the IOP measured in the fellow eye. Use of a prostaglandin analog was the only factor that contributed to the fluctuations in IOP measurements. Therefore, multiple IOP measurements at a single visit may be needed to evaluate the variability of IOP derived from factors associated with the order of IOP measurement and successive IOP measurement. In addition, consideration should be given to the possibility that fluctuation in IOP may be observed during IOP measurements in patients with glaucoma who are treated with prostaglandin analogs.

\section{Author contributions}

KY substantially contributed to the conception and design, acquisition of data, and analysis and interpretation of data, drafted the article or revised it critically for important intellectual content, and agreed to be accountable for all aspects of the work in ensuring that questions related to the accuracy or integrity of any part of the work are appropriately investigated and resolved. AF substantially contributed to the conception and design, acquisition of data, or analysis and interpretation of data. MS, AM, and TF finally approved the version to be published and agreed to be accountable for all aspects of the work in ensuring that questions related to the accuracy or integrity of any part of the work are appropriately investigated and resolved. All authors contributed toward data analysis, drafting and revising the paper and agree to be accountable for all aspects of the work.

\section{Disclosure}

KY received grants from Alcon Japan Ltd. and nonfinancial support from Alcon Japan Ltd., Otsuka Pharmaceutical Co., Ltd., Santen Pharmaceutical Co., Ltd., and Senju Pharmaceutical Co., Ltd; AF received grant from Alcon Japan Ltd. and nonfinancial support from Otsuka Pharmaceutical Co., Ltd. and Santen Pharmaceutical Co., Ltd.; MS received grant from Alcon Japan Ltd. and nonfinancial support from Alcon Japan Ltd. and Pfizer Japan Inc.; AM, none; and TF received grant from Abbott Medical Optics Inc., Otsuka Pharmaceutical Co., Ltd., Kowa Pharmaceutical Company Ltd., Santen Pharmaceutical Co., Ltd., Senju Pharmaceutical Co., Ltd., Alcon Japan Ltd., Retina Kitanihon Co., Ltd., Atsuzawa Proteze Co., Ltd., Shiga Medical Instruments Co., Ltd., and Union Medical Co., Ltd. and nonfinancial support from Abbott Medical Optics Inc., Otsuka Pharmaceutical 
Co., Ltd., Kowa Pharmaceutical Company Ltd., Santen Pharmaceutical Co., Ltd., Senju Pharmaceutical Co., Ltd., Alcon Japan Ltd., Novartis Pharma K.K., Pfizer Japan Inc., Glaucos Japan Co., Ltd., and Abbvie Inc. The authors report no other conflicts of interest in this work.

\section{References}

1. Committee of the Japan Glaucoma Society Guidelines for Glaucoma. The Japan glaucoma society guidelines for glaucoma. 4th eds. Nippon Ganka Gakkai Zasshi. 2018;122:3-53.

2. Goldmann H, Schmidt T. Applanation tonometry. Ophthalmologica. 1957;134(4):221-242.

3. Rosen DA, Johnston VC. Ocular pressure patterns in the Valsalva maneuver. Arch Ophthalmol. 1959;62:810-816.

4. Brody S, Erb C, Veit R, Rau H. Intraocular pressure changes: the influence of psychological stress and the Valsalva maneuver. Biol Psychol. 1999;51(1):43-57.

5. dos Santos MG, Makk S, Berghold A, Eckhardt M, Haas A. Intraocular pressure difference in Goldmann applanation tonometry versus Perkins hand-held applanation tonometry in overweight patients. Ophthalmology. 1998;105(12):2260-2263.

6. Lanigan LP, Clark CV, Hill DW. Intraocular pressure responses to systemic autonomic stimulation. Eye. 1989;3(pt 4):477-483.

7. Bhorade AM, Gordon MO, Wilson B, Weinreb RN, Kass MA; Ocular Hypertension Treatment Study Group. Variability of intraocular pressure measurements in observation participants in the ocular hypertension treatment study. Ophthalmology. 2009;116(4):717-724.

8. Liu JH, Sit AJ, Weinreb RN. Variation of 24-hour intraocular pressure in healthy individuals: right eye versus left eye. Ophthalmology. 2005; 112(10):1670-1675

9. Vernon SA, Jones SJ. Intraocular pressure asymmetry in a population tested with the Pulsair non-contact tonometer. Eye. 1991;5(pt 6):674-677.

10. Dane S, Gümüştekin K, Yazici AT, Baykal O. Correlation between hand preference and intraocular pressure from right- and left-eyes in right- and left-handers. Vision Res. 2003;43(4):405-408.

11. Shuba LM, Doan AP, Maley MK, et al. Diurnal fluctuation and concordance of intraocular pressure in glaucoma suspects and normal tension glaucoma patients. J Glaucoma. 2007;16(3):307-312.

12. Pekmezci M, Chang ST, Wilson BS, Gordon MO, Bhorade AM. Effect of measurement order between right and left eyes on intraocular pressure measurement. Arch Ophthalmol. 2011;129(3):276-281.

13. Gaton DD, Ehrenberg M, Lusky M, et al. Effect of repeated applanation tonometry on the accuracy of intraocular pressure measurements. Curr Eye Res. 2010;35(6):475-479.

14. Moses RA, Liu CH. Repeated applanation tonometry. Am J Ophthalmol. 1968;66(1):89-91
15. Gandhi PD, Gürses-Ozden R, Liebmann JM, Ritch R. Attempted eyelid closure affects intraocular pressure measurement. Am J Ophthalmol. 2001;131(4):417-420.

16. Jamal KN, Gürses-Ozden R, Liebmann JM, Ritch R. Attempted eyelid closure affects intraocular pressure measurement in open-angle glaucoma patients. Am J Ophthalmol. 2002;134(2):186-189.

17. Bright DC, Potter JW, Allen DC, Spruance RD. Goldmann applanation tonometry without fluorescein. Am J Optom Physiol Opt. 1981; 58(12):1120-1126.

18. Goldmann H. Some basic problems of simple glaucoma. Am J Ophthalmol. 1959;48(3):213-220.

19. Grant WM, English FP. An explanation for so-called consensual pressure drop during tonography. Arch Ophthalmol. 1963;69:314-316.

20. Epley KD, Tychsen L, Lueder GT. The effect of an eyelid speculum on intraocular pressure measurement in children. Am J Ophthalmol. 2002; 134(6):926-927.

21. Klein HE, Krohne SG, Moore GE, Mohamed AS, Stiles J. Effect of eyelid manipulation and manual jugular compression on intraocular pressure measurement in dogs. $J$ Am Vet Med Assoc. 2011;238(10): $1292-1295$

22. Kim KS, Kim JM, Park KH, Choi CY, Chang HR. The effect of cataract surgery on diurnal intraocular pressure fluctuation. $J$ Glaucoma. 2009;18(5):399-402.

23. Klink T, Praetorius S, Leippi S, Klink J, Grehn FJ. Diurnal and nocturnal intraocular pressure fluctuations after trabeculectomy. Ophthalmologica. 2012;227(3):160-165.

24. Peplinski LS, Albiani Smith K. Deepening of lid sulcus from topical bimatoprost therapy. Optom Vis Sci. 2004;81(8):574-577.

25. Shah M, Lee G, Lefebvre DR, et al. A cross-sectional survey of the association between bilateral topical prostaglandin analogue use and ocular adnexal features. PLoS One. 2013;8(5):e61638.

26. Lee YK, Lee JY, Moon JI, Park MH. Effectiveness of the ICare rebound tonometer in patients with overestimated intraocular pressure due to tight orbit syndrome. Jpn J Ophthalmol. 2014;58(6):496-502.

27. Lee GA, Ritch R, Liang SY, et al. Tight orbit syndrome: a previously unrecognized cause of open-angle glaucoma. Acta Ophthalmol. 2010; 88(1):120-124

28. Na JI, Kwon OS, Kim BJ, et al. Ethnic characteristics of eyelashes: a comparative analysis in Asian and Caucasian females. Br J Dermatol. 2006;155(6):1170-1176.

29. Park DH, Choi WS, Yoon SH, Song CH. Anthropometry of asian eyelids by age. Plast Reconstr Surg. 2008;121(4):1405-1413.

30. Phelps CD, Phelps GK. Measurement of intraocular pressure: a study of its reproducibility. Albrecht Von Graefes Arch Klin Exp Ophthalmol. 1976;198(1):39-43.

31. Dielemans I, Vingerling JR, Hofman A, Grobbee DE, de Jong PT. Reliability of intraocular pressure measurement with the Goldmann applanation tonometer in epidemiological studies. Graefes Arch Clin Exp Ophthalmol. 1994;232(3):141-144.
Clinical Ophthalmology

\section{Publish your work in this journal}

Clinical Ophthalmology is an international, peer-reviewed journal covering all subspecialties within ophthalmology. Key topics include: Optometry; Visual science; Pharmacology and drug therapy in eye diseases; Basic Sciences; Primary and Secondary eye care; Patient Safety and Quality of Care Improvements. This journal is indexed on Submit your manuscript here: http://www.dovepress.com/clinical-ophthalmology-journal
Dovepress

PubMed Central and CAS, and is the official journal of The Society of Clinical Ophthalmology (SCO). The manuscript management system is completely online and includes a very quick and fair peer-review system, which is all easy to use. Visit http://www.dovepress.com/ testimonials.php to read real quotes from published authors. 